LAWRENCE LIVERMORE NATIONAL LABORATORY

\title{
The National Ignition Facility High-Energy Ultraviolet Laser System
}

E. I. Moses

September 15, 2003

$3^{\text {rd }}$ International Symposium on Lasers \& Nonlinear Materials, Keystone, Colorado, July 20-24, 2003 
This document was prepared as an account of work sponsored by an agency of the United States Government. Neither the United States Government nor the University of California nor any of their employees, makes any warranty, express or implied, or assumes any legal liability or responsibility for the accuracy, completeness, or usefulness of any information, apparatus, product, or process disclosed, or represents that its use would not infringe privately owned rights. Reference herein to any specific commercial product, process, or service by trade name, trademark, manufacturer, or otherwise, does not necessarily constitute or imply its endorsement, recommendation, or favoring by the United States Government or the University of California. The views and opinions of authors expressed herein do not necessarily state or reflect those of the United States Government or the University of California, and shall not be used for advertising or product endorsement purposes. 


\title{
The National Ignition Facility High-energy Ultraviolet Laser System
}

\author{
Edward I. Moses \\ Lawrence Livermore National Laboratory, P.O. Box 808 L-466, Livermore, CA 94551
}

\begin{abstract}
The National Ignition Facility (NIF), currently under construction at the Lawrence Livermore National Laboratory, is a stadium-sized facility containing a 192-beam, 1.8-Megajoule, 500-Terawatt, ultraviolet laser system together with a 10-meter diameter target chamber with room for nearly 100 experimental diagnostics. When completed, NIF will be the world's largest and most energetic laser experimental system, providing an international center to study inertial confinement fusion and the physics of matter at extreme energy densities and pressures. NIF's 192 energetic laser beams will compress fusion targets to conditions required for thermonuclear burn, liberating more energy than required to initiate the fusion reactions. Other NIF experiments will allow the study of physical processes at temperatures approaching $10^{8} \mathrm{~K}$ and $10^{11} \mathrm{Bar}$, conditions that exist naturally only in the interior of stars, planets and in nuclear weapons. NIF is now entering the first phases of its laser commissioning program. The first four beams of the NIF laser system have generated 106 kilojoules of infrared light and over $10 \mathrm{~kJ}$ at the third harmonic $(351 \mathrm{~nm})$. NIF's target experimental systems are also being installed in preparation for experiments to begin in late 2003. This paper provides a detailed look the NIF laser systems, the significant laser and optical systems breakthroughs that were developed, the results of recent laser commissioning shots, and plans for commissioning diagnostics for experiments on NIF.
\end{abstract}

C 2003 Elsevier Science B.V. All rights reserved.

PACS: 42.60.-v; 42.60.By; 42.60.Da; 42.60.Jf; 42.60.Lh; 42.62.-b; 42.65.Ky

Keywords: Solid-state Laser; Inertial Confinement Fusion; High Energy Density Physics; Neodymium-doped glass laser

\section{Introduction}

The National Ignition Facility (NIF) under construction at the Lawrence Livermore National Laboratory (LLNL) will be a U. S. Department of Energy and National Nuclear Security Administration (NNSA) national center to study inertial confinement fusion and the physics of extreme energy densities and pressures. Construction of the building that houses the laser system was completed in September 2001 and the construction of all 192 ultra-clean and precision aligned beam path enclosures was completed in September 2003. In late 2002 NIF began activating its first four laser beam lines. By July 2003 NIF had delivered world-record single laser energy performance in primary, second, and third harmonic wavelengths (NIF's primary wavelength is 1.06 micron infrared light). When completed in 2008, NIF will provide up to 192 energetic laser beams to compress deuterium-tritium fusion targets to conditions where they will ignite and burn, liberating more energy than is required to initiate the fusion reactions. NIF experiments will allow the 


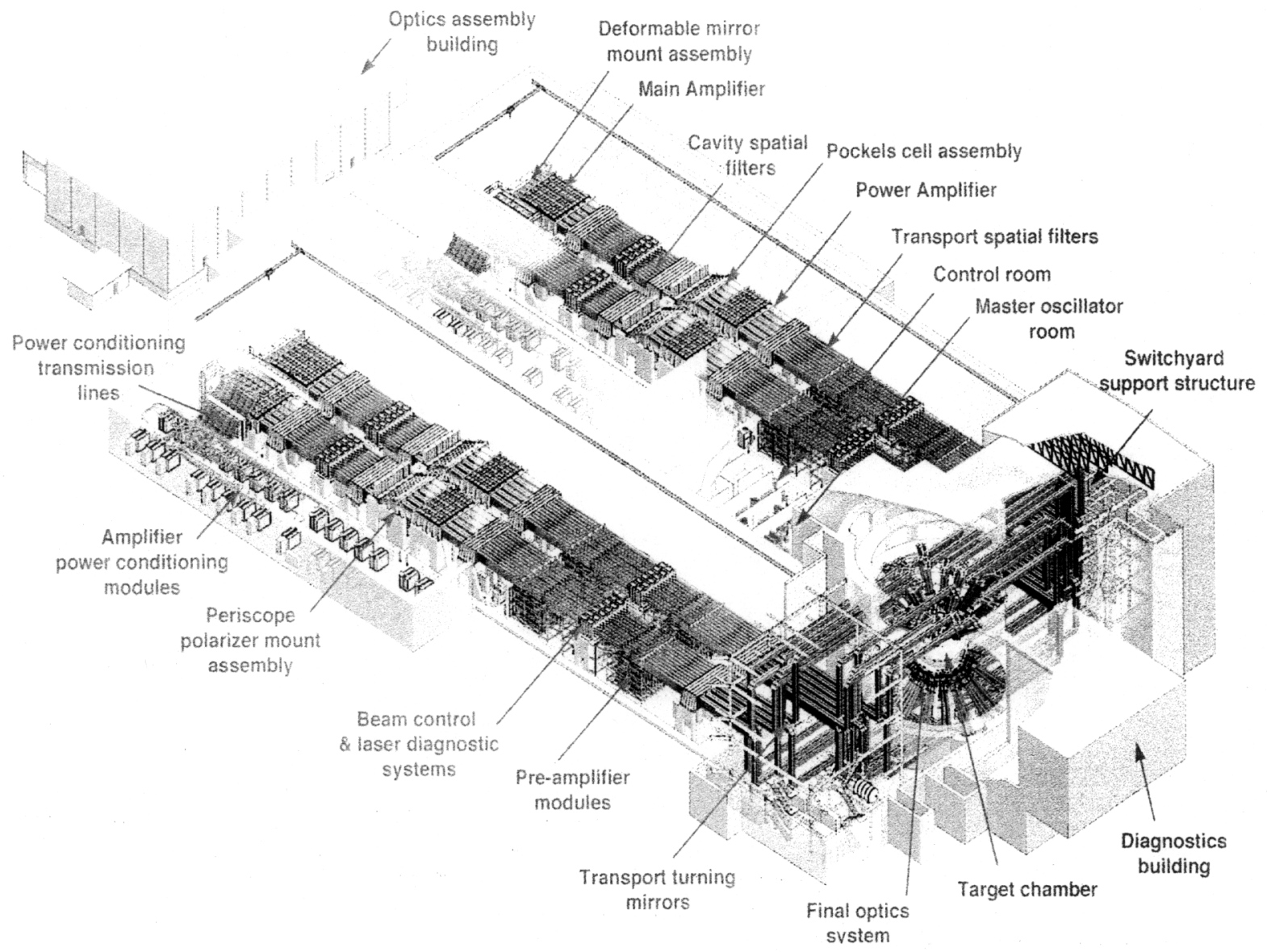

Fig. 1. Schematic view of the National Ignition Facility showing the main elements of the laser system. The 10meter diameter target chamber sets the scale for the facility.

study of physical processes at temperatures approaching 100 million $\mathrm{K}$ and 100 billion times atmospheric pressure. These conditions exist naturally only in the interior of stars and in nuclear weapons explosions [1-4].

\section{A Description of NIF}

The National Ignition Facility layout is shown in Figure 1. [9] NIF consists of a number of subsystems including amplifier power conditioning modules to drive large flashlamp arrays, the injection laser system consisting of the master oscillator and preamplifier modules, the main laser system along with its optical components, the switchyards, and the 10-meter diameter target chamber and its experimental systems. The entire laser system, switchyards, and target area is housed in an environmentally controlled building. An integrated computer control system is located in the core of the facility to monitor, align, and operate the more than 60,000 control points re- quired for NIF's operation. A 2,000 square meter cleanroom facility, the Optics Assembly Building is located at one end of NIF for assembling and installing the precision optical and optomechanical components that make up the NIF laser system. On the opposite end of the facility the Diagnostics Building houses experimenters, a data acquisition system, and target preparation and storage areas.

NIF's laser system is comprised of 192 highenergy laser beams. For inertial fusion studies the laser beams will produce a nominal 1.8 million joules (approximately 500 trillion watts of power for 3 nanoseconds) of laser energy in the third harmonic $(3 \omega$, or 351 nanometer wavelength) onto a target. This is approximately 50 times the energy available in the Nova laser, which was operated at LLNL between 1983 and 1999 or the Omega Laser at the University of Rochester's Laboratory for Laser Energetics. NIF is capable of providing a range of beam energies and powers for experimental and diagnostic $\mathrm{x}$-ray backlighter applications. 


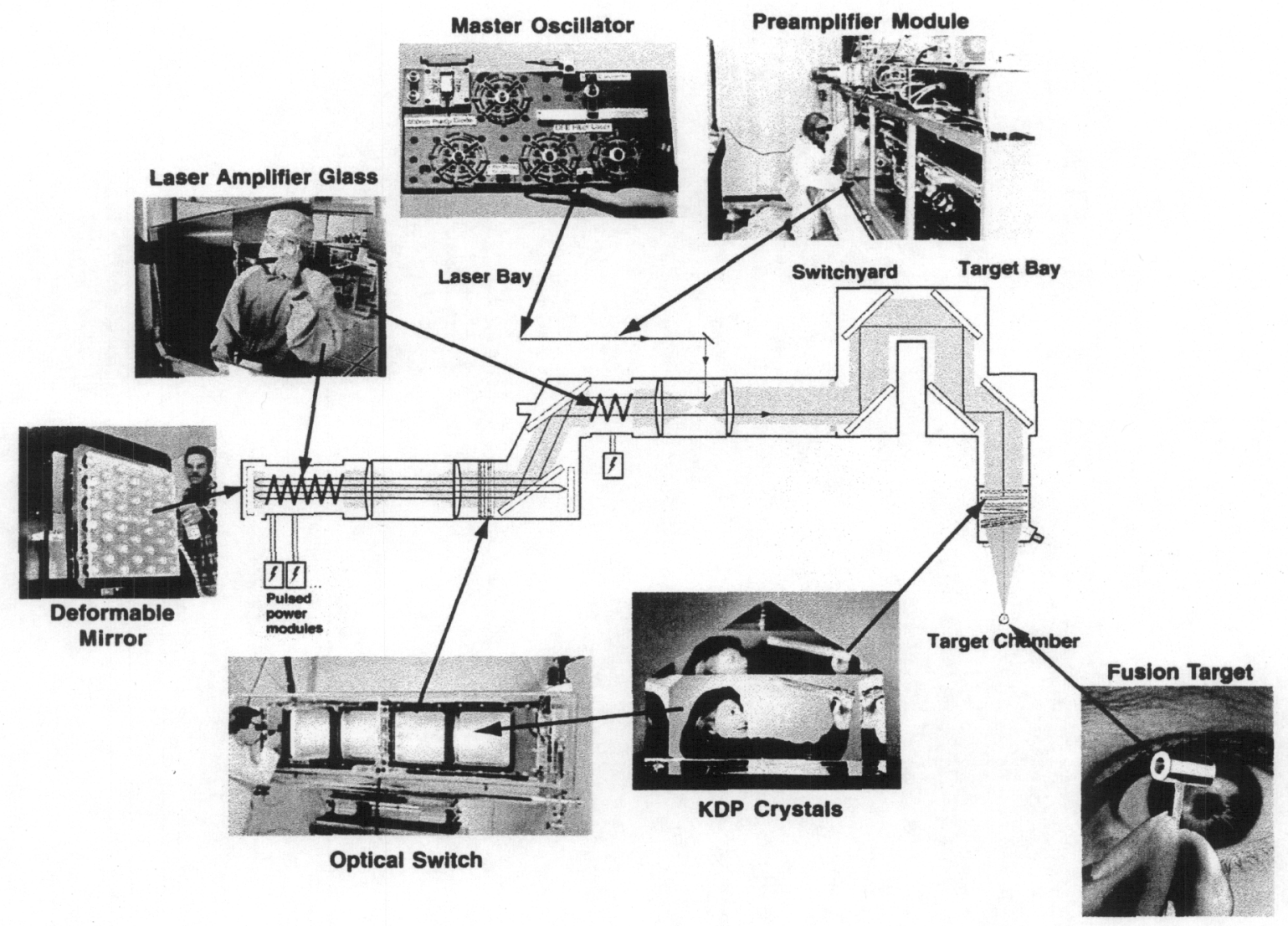

Fig. 2. Schematic representation of a NIF laser beam line highlighting some of the key technology developments.

Figure 2 schematically shows one of the 192 laser beams, detailing the key elements of a NIF beamline. A NIF laser beam begins with a very modest nanojoule energy pulse from the master oscillator and a diode-pumped fiber amplifier system that can provide a variety of pulse shapes suitable for a wide range of experiments, from complex, high-contrast temporally shaped pulses for ICF implosions, to high-energy extended time pulses. The master oscillator pulse is transported to preamplifier modules (PAMs) for amplification and beam shaping. Each PAM first amplifies the pulse by a factor of one million (to about one millijoule) using a diode-pumped fiber laser system and then boosts the pulse once again by a factor of 20,000 , this time to a maximum of 10 joules, by passing the beam four times through a neodymium-doped glass flashlamp-pumped amplifier. When completed, there will be a total of 48 PAMs on NIF, each feeding a "quad" of four laser beams.

From the PAM the laser beam next enters the main laser system, which consists of two large amplifier units - the power amplifier, and the multi-pass or main amplifier. These amplifier systems are designed to efficiently amplify the few joule input pulse from the PAM to the re- quired energy and power, maintaining the input beam's spatial, spectral, and temporal characteristics. The amplifiers use 3,072 40 kilogram slabs, measuring $46 \mathrm{~cm} \times 81 \mathrm{~cm} \times 3.4 \mathrm{~cm}$, of neodymium-doped phosphate glass set vertically on edge at Brewster's angle to minimize reflective losses in the laser beam [9]. The slabs are stacked four high and two wide to accommodate a "bundle" of eight laser beams. The amplifiers, with 16 glass slabs per beam, are arranged with 11 slabs in the main amplifier section and five slabs in the power amplifier section (the power amplifier can actually accommodate 7 slabs per beam if necessary for future applications). Together, even though of relatively low gain $\left(\sim 10^{4}\right)$, these amplifiers provide $99.9 \%$ of NIF's power and energy.

The slabs are surrounded by vertical arrays of flashlamps, measuring $180 \mathrm{~cm}$ in length. A total of 7680 flashlamps are required for NIF's 192 laser beams. Each flashlamp is driven by 30,000 joules of electrical energy. The intense white light from the flashlamps excites the neodymium in the laser slabs to provide optical gain at the primary wavelength of the laser. Some of the energy stored in the neodymium is released when the laser beam passes through the slab. Advances in glass amplifier technology allow NIF to operate 
with less than twice the number of flashlamps than Nova even though the laser system will produce up to 60 times more output energy. The flashlamps are cooled between shots, along with the amplifier slabs, using chilled synthetic air. Operational experience has now shown that it is possible to fire the NIF laser system as often as once every 4 hours. A recent shot campaign provided three shots per day over a three-day period, demonstrating the ability to meet the planned 700 shots per year when NIF is fully operational.

The NIF amplifier flashlamps receive their power from the Power Conditioning System (PCS), which consists of the highest energy array (delivering up to $\sim 400$ megajoules) of electrical capacitors ever assembled. The PCS occupies four capacitor bays adjacent to each laser bay as shown in Fig. 1. Each PCS module is configured with eight, 20-capacitor modules delivering 1.7 megajoules per module that power the flashlamps for one beam. Continuous testing on a dedicated PCS and flashlamp test system have now fired over 19000 times at a rate of 1200 shots per month, corresponding to nearly the entire NIF projected 30-year lifetime. Nine power conditioning modules are now installed and reliably delivering electrical power to flashlamps in Laser Bay 2 for the first four laser beams [9].

A key component in each beam line is an optical switch called a plasma-electrode Pockels cell (PEPC). The PEPC uses electrically induced changes in the refractive index of a thin plate of electro-optic crystal, made of potassium dihydrogen phosphate (KDP) sandwiched between two gas-discharge plasmas. The plasma density is approximately $10^{12} / \mathrm{cm}^{3}$ and there is no effect on the laser beam passing through the cell, yet the plasmas form sufficiently conducting electrodes, allowing the entire surface of the thin crystal plate to charge electrically in about 100 nanoseconds so the entire beam can be switched efficiently. When combined with a polarizer, the PEPC allows light to pass through or reflect off the polarizer. The PEPC traps the laser light between two mirrors as it makes four one-way passes through the main amplifier system before being switched out to continue its way to the target chamber.

All major laser components are assembled in clean, pre-aligned modules called line-replaceable units or LRUs. These LRUs contain laser optics, mirrors, lenses, and hardware such as pinhole filter assemblies that are designed to be robotically installed into NIF's beampath infrastructure, while maintaining the high level of cleanliness required for proper laser operation. Automated guided vehicles carrying portable clean rooms position themselves underneath NIF's beampath enclosures and robotically insert LRU's into the beampath. The installation, integration, and commissioning of the beampath infrastructure at the required cleanliness levels has been successfully accomplished for the more than 120 LRU's required for NIF first four laser beamlines.

NIF's 10 meter diameter target chamber includes a number of laser entry ports that allow quads of four laser beams to be focused to the center of the target chamber through a final optics assembly (FOA). The FOA is a precision optical assembly containing optics to provide a variety of beam profiles on target, KDP and deuterated KDP plates to convert the infrared laser light into the ultraviolet, the final focus lens, debris shields and vacuum gate valve for each beam.

NIF uses over 7500 large optics, including glass slabs, KDP crystals, mirrors, windows, lenses, polarizers, and diffraction gratings. There are over 26,000 smaller optics used in the Injection Laser System. [10,11]

The NIF target chamber and final focusing system has been designed with maximum flexibility for experimental users with 120 diagnostic instrumentation and target insertion ports. During initial operation, NIF is configured to operate in the "indirect drive" configuration, which directs the laser beams into two cones in the upper and lower hemispheres of the target chamber. This configuration is optimized for illuminating the fusion capsule mounted inside cylindrical hohlraums using $x$-rays generated from the hot walls of the hohlraum to implode the capsule. NIF can also be configured in a "direct drive" arrangement of beams, by moving some quads of beams from the upper and lower hemispheres into a more symmetric arrangement of beams. Direct drive ignition requires better energy and power balance between laser beams and better beam smoothing and focusing but some think this configuration may ultimately be more attractive for producing a viable power production plant because of the higher gain available. [5] 

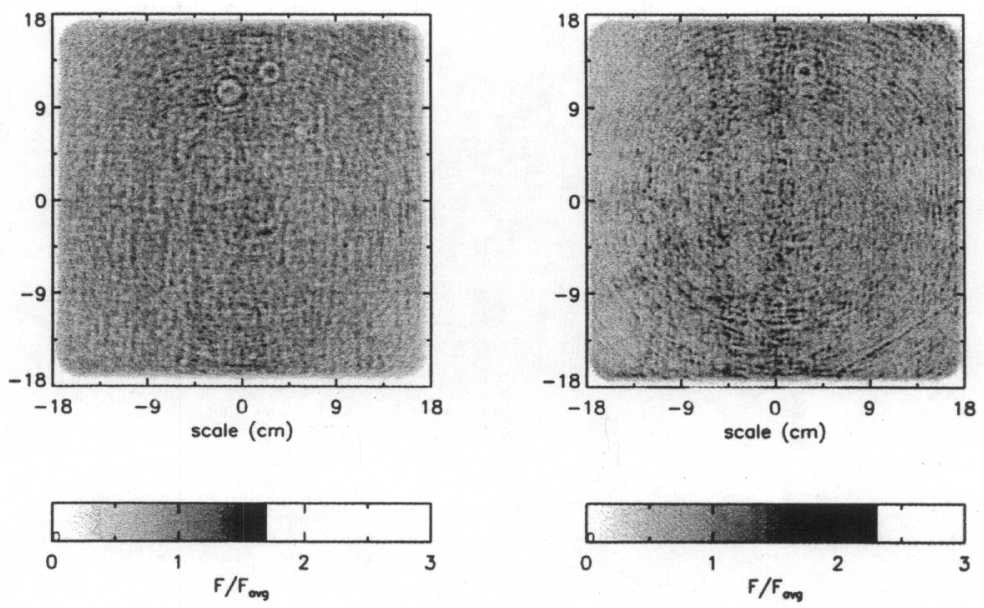

Fig. 3 Near field image of an $11.4 \mathrm{~kJ} 2 \omega$ and $10.4 \mathrm{~kJ} 3 \omega$ NIF beams showing excellent contrast uniformity.

\section{NIF Project Status}

NIF construction began in May 1997 and nearly all 192 beampath enclosures are now in place and ready for optics installation. In October 2001 the first laser light from NIF's master oscillator was generated in the master oscillator room located in the central core of the NIF building. This master oscillator has demonstrated the required pulse shaping stability and accuracy for high contrast ignition pulses and other types of laser pulses that are of interest to NIF experimenters. In June 2002 the first preamplifier module was installed in the Laser Bay and routinely amplifies master oscillator pulses to the joule level.

First high energy ultraviolet laser light to the center of NIF's target chamber was achieved in January 2003 with approximately 1 kilojoule (kJ) of laser energy focused onto a simple foil target. The energetic $\mathrm{x}$-rays emitted from this target were measured with an $\mathrm{x}$-ray pinhole imaging system called the Static X-ray Imager (SXI) mounted on the target chamber. In April $200310.6 \mathrm{~kJ}$ of ultraviolet light was produced in four beams and directed to a target in the target chamber.

A separate target chamber, known as the Precision Diagnostic System (PDS) is being used to fully characterize NIF's laser performance. Any one of the four activated NIF beams can be directed into the PDS using a special robotic mirror and transport system (eventually the PDS will be used to study all 96 individual beams of Laser Bay 2). Data from the PDS is being used to validate and enhance computer models used to pre- dict laser performance. A series of laser energy and power performance campaigns have been carried out using PDS to characterize $1 \omega, 2 \omega$, and $3 \omega$ performance. Figure 3 shows examples of high energy $2 \omega$ and $3 \omega$ beams imaged in the near field using the PDS.

At this time NIF's highest $3 \omega$ single laser beam performance is $10.4 \mathrm{~kJ}$, equivalent to $2 \mathrm{MJ}$ for a fully activated NIF, exceeding the NIF energy point design of $1.8 \mathrm{MJ}$. The $10.4 \mathrm{~kJ} 3 \omega$ energy was achieved with $13.65 \mathrm{~kJ} 1 \omega$ drive in a 3.5 ns pulse. Also during this time a series of shots were conducted generating green or $2 \omega$ laser light with single beam energy up to $11.4 \mathrm{~kJ}$ in a $5 \mathrm{~ns}$ square pulse. This is equivalent to nearly 2.2 million Joules (MJ) on target for 192 beams. In July $2003,26.5 \mathrm{~kJ}$ of infrared light per beam was produced. This energy is greater than $30 \%$ of maximum $1 \omega$ single beam drive energy required for NIF. NIF has now demonstrated the highest energy $1 \omega, 2 \omega$, and $3 \omega$ beamlines in the world.

High power shot campaigns have also been completed with drive power reaching 7 terawatts or about 5 gigawatts $/ \mathrm{cm}^{2}$. Figure 4 details energy and power achieved on a number of $1 \omega$ shots conducted through July 2003.

Additional laser performance shots have verified beam-to-beam timing of better than 6 picoseconds as shown in Fig. 5 using measurements taken by the Streaked X-ray Detector diagnostic, demonstrating the ability to control the laser pulse to about 1 part in 150,000 through the total laser beam path. 


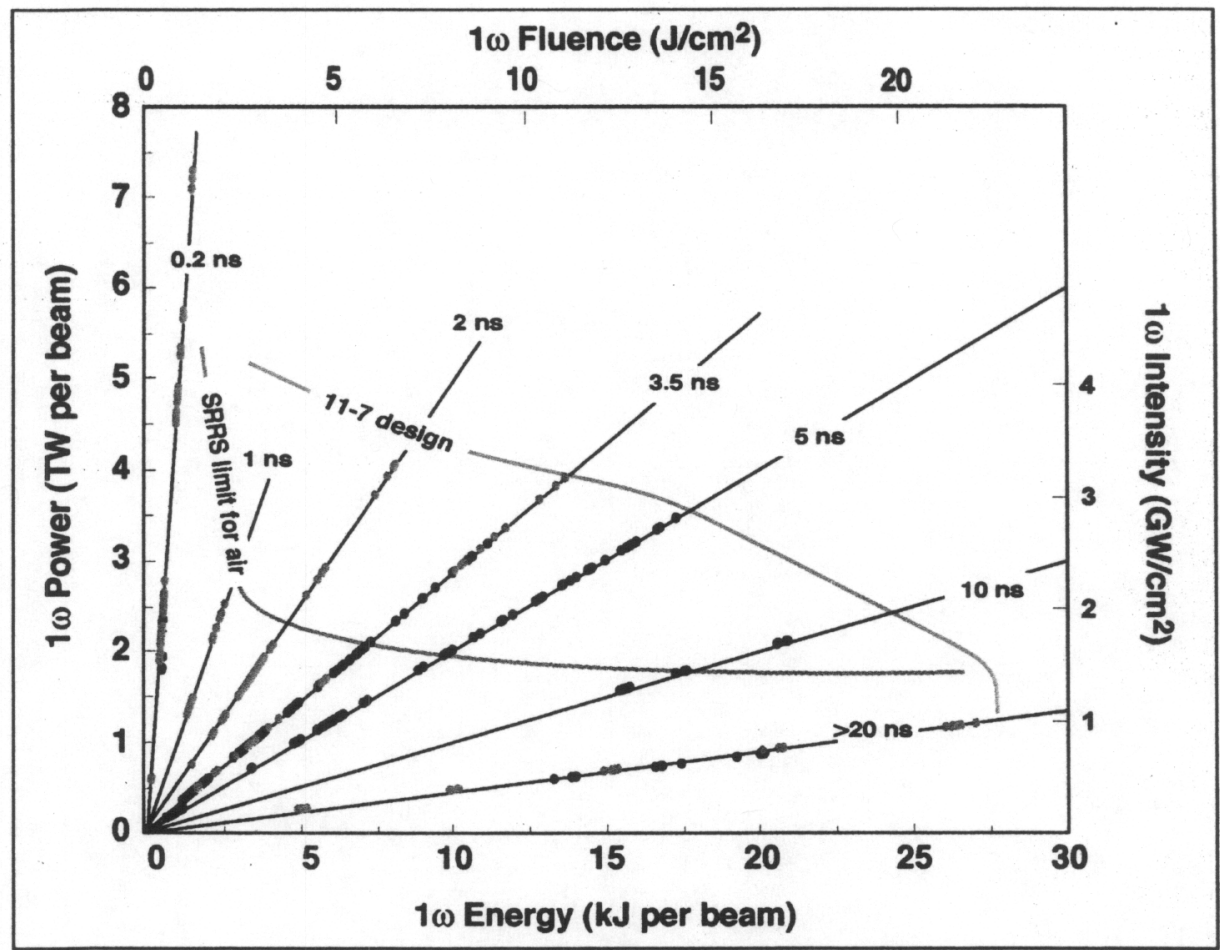

Fig. $41 \omega$ energy versus power is plotted here for a number of NIF performance shots. The plot also indicates the level where Raman scattering limits beam propagation in air - NIF beams propagate in argon gas-filled beam tubes above this limit.

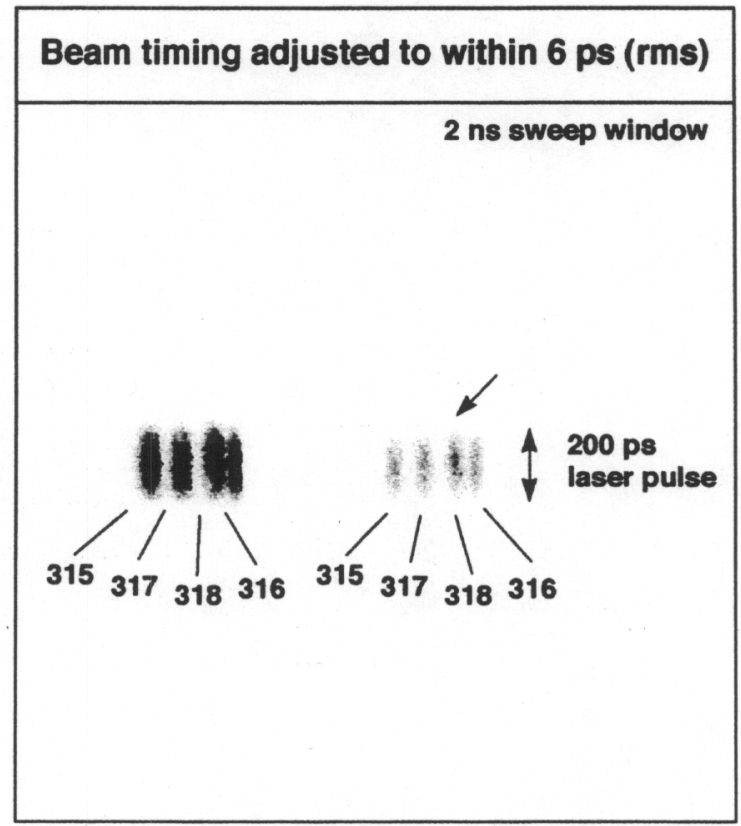

Fig. 5 The Streaked X-ray Detector diagnostic mounted on the NIF target chamber was used to acquire these images of NIF beams. The individual beams are shown in the closely spaced groups of four vertical streaks. Different thickness filters are placed in front of imaging slits to give a measure of the $\mathrm{x}$-ray energy, with a thicker filter placed in the right hand slit, which gives the dimmer group of beam streaks shown on the right. 

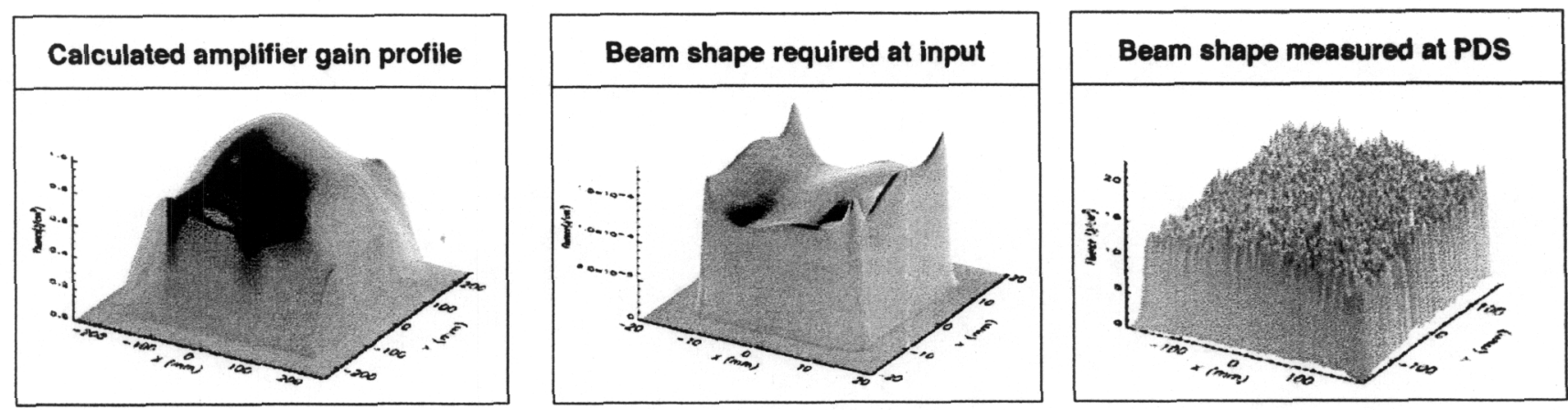

Fig. 6 shows the modeled amplifier gain profiles, and compensated beam shape at the input to the preamplifier along with a resulting measured beam profile.

Beam uniformity on NIF at $1 \omega$ is required to be $10 \%$ or better. In order to achieve the required uniformity special compensation is used in the Injection Laser System PAMs. Compensation corrects the non-uniform gain profiles in the large glass amplifier system by the application of spatial shaping in the pre-amplifier module. A combination of serrated apertures and patterned gain compensation masks are used. Figure 6 shows the modeled amplifier gain profiles, and compensated beam shape at the input to the preamplifier along with a resulting measured beam profile demonstrating that this compensation meets NIF's requirements for uniformity and flatness. $1 \omega$ farfield divergence is measured to be smaller than $80 \%$ energy contained within $18 \mu$ radians required for NIF. Figure 7 shows examples of model predictions and measurements.
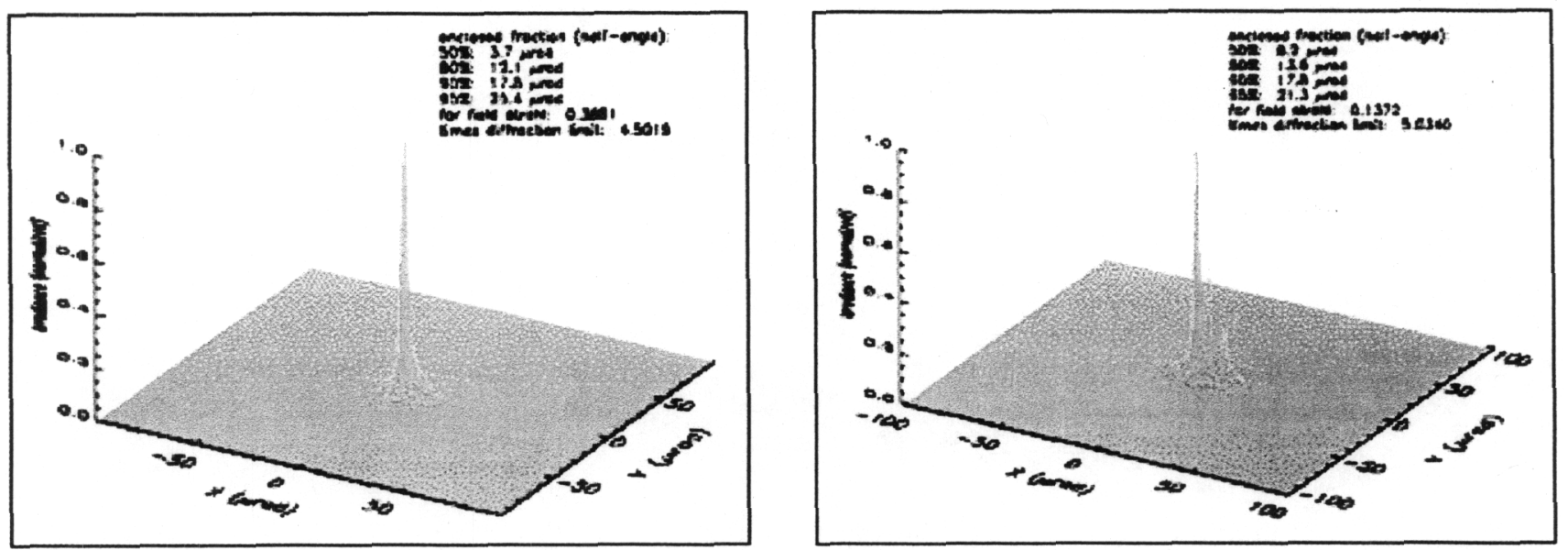

Fig. 7 shows $1 \omega$ far-field divergence modeled with the NIF laser propagation code PROP compared with actual data. The model predicts $80 \%$ energy contained within $12.1 \mu \mathrm{rad}$, compared to the measures $13.6 \mu \mathrm{rad}$. NIF requires that $80 \%$ of the laser energy be contained within $18 \mu \mathrm{rad}$. 


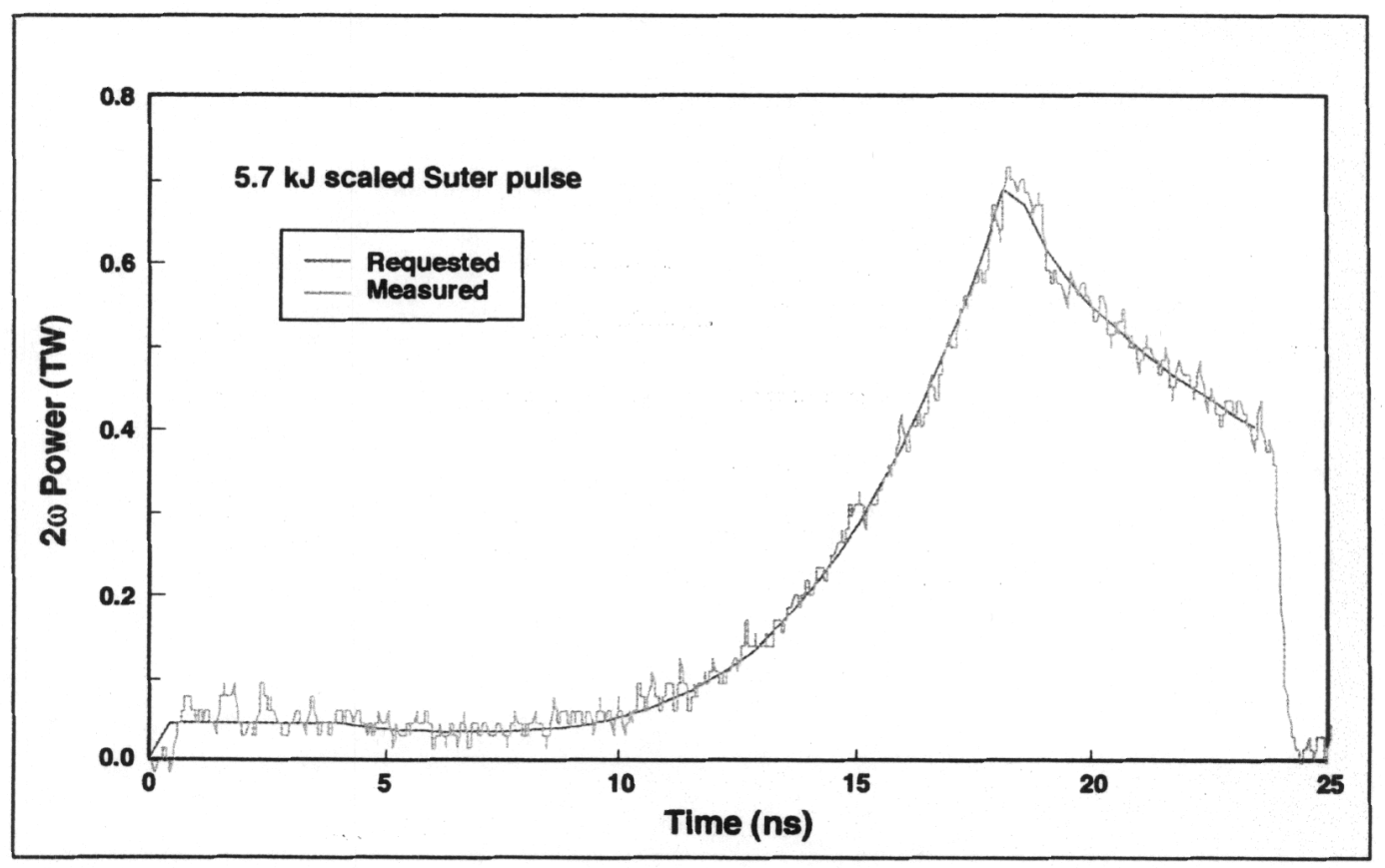

Fig. 8 An example of a shaped $2 \omega$ pulse with total energy of $5.7 \mathrm{~kJ}$ and pulse length of approximately $25 \mathrm{~ns}$.
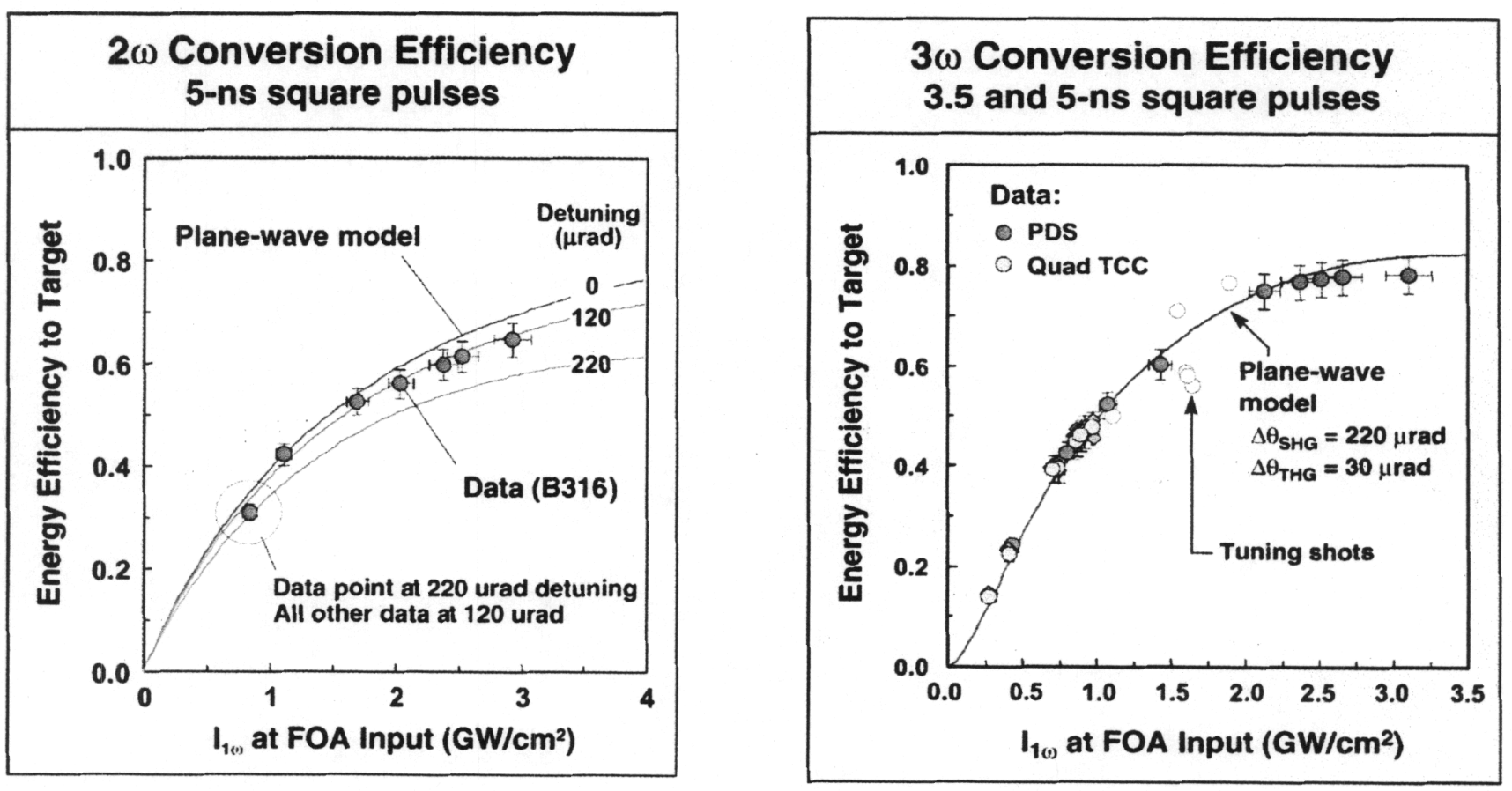

Fig. $9 \mathrm{a}$ on the left and $9 \mathrm{~b}$ on the right shows measured $2 \omega$ and $3 \omega$ conversion efficiency, respectively, for a number of input powers. Overlaid on the data points is a plane-wave model that compares will with the measurements. 
Temporal shaping is an important capability on NIF for meeting experimental needs. Figure 8 shows an example of a shaped $2 \omega$ pulse with total energy of $5.7 \mathrm{~kJ}$ and pulse length of approximately $25 \mathrm{~ns}$. The measured pulse shape is overlaid on top of the requested shape showing NIF's capability for delivering prescribed pulse shaping. This particular pulse is of interest for ignition experiments to be conducted when NIF is completed.

Frequency conversion efficiency is important for maximizing the energy available for experiments. We have conducted studies to measure the conversion efficiency of the KDP doubler and deuterated KDP tripler crystals in the final optics system. Figures $9 a$ and $b$ show data and simulations for $2 \omega$ conversion efficiency measured on the PDS. The data shows that $2 \omega$ and $3 \omega$ conversion efficiency can provide $>2 \mathrm{MJ}$ full NIF equivalent energy.

\section{Conclusions and Future Directions}

Completion of all 192 laser beams is scheduled for September 2008. In the time between first light and project completion, approximately 1500 experiments are planned in support of the Stockpile Stewardship Program, inertial confinement fusion, high energy density physics, weapons effects, inertial fusion energy, and basic science [5-8] After project completion, NIF is expected to provide approximately 700 shots per year for a wide variety of experimental users as a national user facility.

The first physics experiments are already being performed on NIF. [12] Initial experiments are studying laser-plasma interactions and hydrodynamics of shocked materials. In the coming year this unique facility will already be providing the first glimpses of conditions heretofore only found in the most extreme environments. This will be done under repeatable and wellcharacterized laboratory conditions for the benefit of basic and applied science. [13]

\section{Acknowledgments}

The author would like to express his appreciation to the many people, institutions, and industrial partners that are diligently working to construct the National Ignition Facility. This work was performed under the auspices of the U.S. Department of Energy by the University of Califor- nia, Lawrence Livermore National Laboratory under contract W-7405-Eng-48.

\section{References}

[1] J. Lindl, Inertial Confinement Fusion: The Quest for Ignition and Energy Gain Using Indirect Drive, Springer-Verlag (1998).

[2] "Laboratory Microfusion Capability Phase-II Study," prepared by Interscience, Inc. for the Inertial Fusion Division Office of Research and Advanced Technology, ISI-TM9005281, May 31, 1990.

[3] "Solid State Lasers for Application to Inertial Confinement Fusion (ICF)," W. F. Krupke, ed., Proc. SPIE 2633 (1995).

[4] C. B. Tarter, "Inertial Fusion and Higher Energy Density Science in the United States," Proc. 2001 Conf. On Inertial Fusion Science and Applications (IFSA 2001), K. A. Tanaka, D. D. Meyerhofer, J. Meyer-terVehn, eds., Elsevier (2002).

[5] S. Haan, et al., "Update On Target Design for the National Ignition Facility," Proc. 2001 Conf. On Inertial Fusion Science and Applications (IFSA 2001), K. A. Tanaka, D. D. Meyerhofer, J. Meyer-ter-Vehn, eds., Elsevier (2002).

[6] B. Remington, "High Energy Density Astrophysics in the Laboratory," Proc. 2001 Conf. On Inertial Fusion Science and Applications (IFSA 2001), K. A. Tanaka, D. D. Meyerhofer, J. Meyer-ter-Vehn, eds., Elsevier (2002).

[7] "High-Energy-Density Physics Study Report," prepared by the National Nuclear Security Administration Office of Defense Programs, April 6, 2001.

[8] E. Moses, et al., "The National Ignition Facility: Status and Plans for Laser Fusion and High-Energy-Density Experimental Studies," Fusion Science and Technology, V. 43, p. 420, May 2003.

[9] M. A. Newton, et al., "Initial Activation and Operation of the Power Conditioning System for the National Ignition Facility," Proceedings of the International Pulsed Power Conference 2003, Dallas, TX, June 15-18, 2003.

[10] E. Moses, et al., "The National Ignition Facility: The World's Largest Optics and Laser System," UCRL-151593-JC, submitted for publication in the SPIE Proc. Of Photonics West, January 2003. 
[11] C. J. Stolz, et al., "Fabrication of meter-scale laser resistant mirrors for the National Ignition Facility, a fusion laser," submitted to the SPIE Proceddings of the International Symposium on Optical Science and Technology, Advances in Mirror Technology for X-ray, EUV Lithography, Laser, and Other Applications, 2003.

[12] S. Glenzer, et al., "Progress in Long Scale Length Laser Plasma Interactions," submitted to the Proceedings of the Third International Conference on Inertial Fusion Sciences and Applications, Monterey, CA, September 2003.

[13] For more information on the NIF Project please visit our web site at http://www.llnl.gov/nif 